\title{
Experiences with Extra-Vehicular Activities in Response to Critical ISS Contingencies
}

\author{
E. A. Van Cise ${ }^{1}$, B. J. Kelly², and J. P. Radigan ${ }^{3}$, C. W. Cranmer ${ }^{4}$
}

NASA Lyndon B. Johnson Space Center, Houston, Texas, 77058

\begin{abstract}
The maturation of the International Space Station (ISS) design from the proposed Space Station Freedom to today's current implementation resulted in external hardware redundancy vulnerabilities in the final design. Failure to compensate for or respond to these vulnerabilities could put the ISS in a posture to where it could no longer function as a habitable space station. In the first years of ISS assembly, these responses were to largely be addressed by the continued resupply and Extra-Vehicular Activity (EVA) capabilities of the Space Shuttle. Even prior to the decision to retire the Space Shuttle, it was realized that ISS needed to have its own capability to be able to rapidly repair or replace external hardware without needing to wait for the next cargo resupply mission.

As documented in a previous publicatoin ${ }^{5}$, in 2006 development was started to baseline ExtraVehicular Activity (EVA, or spacewalk) procedures to replace hardware components whose failure would expose some of the ISS vulnerabilities should a second failure occur. This development work laid the groundwork for the onboard crews and the ground operations and engineering teams to be ready to replace any of this failed hardware. In 2010, this development work was put to the test when one of these pieces of hardware failed.

This paper will provide a brief summary of the planning and processes established in the original Contingency EVA development phase. It will then review how those plans and processes were implemented in 2010, highlighting what went well as well as where there were deficiencies between theory and reality. This paper will show that the original approach and analyses, though sound, were not as thorough as they should have been in the realm of planning for next worse failures, for documenting Programmatic approval of key assumptions, and not pursuing sufficient engineering analysis prior to the failure of the hardware.

The paper will further highlight the changes made to the Contingency EVA preparation team structure, approach, goals, and the resources allocated to its work after the 2010 events. Finally, the authors will overview the implementation of these updates in addressing failures onboard the ISS in 2012, 2013, and 2014. The successful use of the updated approaches, and the application of the approaches to other spacewalks, will demonstrate the effectiveness of this additional work and make a case for putting significant time and resources into pre-failure planning and analysis for critical hardware items on humantended spacecraft.
\end{abstract}

\footnotetext{
${ }^{1}$ Flight Director, Flight Director Office, 2101 NASA Parkway / CA8, Houston, TX, 77058, AIAA Member

${ }^{2}$ Research Integration Manager, ISS Research Integration Office, 2101 NASA Parkway / OZ6, Houston, TX, 77058

${ }^{3}$ Flight Director, Flight Director Office, 2101 NASA Parkway / CA8, Houston, TX, 77058

${ }^{4}$ Mission Evaluation Room Manager, On-Orbit Engineering Office, 2101 NASA Parkway / OB2, Houston, TX, 77058

${ }^{5}$ Smith, L. N. and Van Cise, E. A., "Contingency Planning for Removal and Replacement of Critical Equipment During Stage Extra-Vehicular Activity,” AIAA-2008-3287 SpaceOps 2008 Conference
} 